\title{
The Train Dynamics of Wheel Rail Contact and Longitudinal Lateral Interaction between Vehicles
}

\author{
L. Cantone, D. Negretti and V. Vullo \\ Department of Mechanical Engineering \\ University of Rome "Tor Vergata", Italy
}

\begin{abstract}
This paper is focused on the vehicle dynamics caused by the forces exchanged, through buffers and draw gears, by consecutive vehicles on a curve. The results have been obtained by adding a buffers/draw gears contact model on an existing multibody code, previously developed by the authors. The multibody code manages rigid bodies connected by elastic and rigid constraints; the wheel/rail contact model is three dimensional and employs an elastic constraint among wheel and rail. The wheel/rail contact is managed by means of a numerical model called TOAM (third order approximation method). Numerical tests and experimental validations of the proposed model are here presented, considering a train made by three vehicles, running on an $\mathrm{S}$ shaped curve, subjected to parametric compressive forces.
\end{abstract}

Keywords: train dynamics, wheel rail contact, buffers contact, longitudinal forces, derailment, train simulator.

\section{Introduction}

Safety and efficiency of train transportation are more and more requested by the railway operators. In order to provide a reliable and flexible tool to study the dynamics of a train and to reduce the expensive experimental tests, a new numerical model have been proposed by the author [1-3].

In those previous papers, an original multibody software, capable to manage the dynamics of any number of rigid bodies (each one with six degrees of freedom dofs-) coupled by elastic or rigid joints, has been developed. Moreover, the developed code considers a three-dimensional contact between the surfaces of wheel and rail, by considering also an elastic interaction among them.

The further development that has been introduced in this paper is the adding of a new module, which integrates the buffers contact in the multibody code; by this way, the multibody dynamics of three consecutive vehicles, subjected to 
longitudinal compressive forces (LCF), are investigated by computing the contact forces exchanged by the buffer heads of adjacent vehicles, running on a 3D track. Not only the normal contact forces but also the friction forces between the buffer heads are considered in the proposed model; the latter forces grow up due to the relative movements of wagons, during the running on a curve.

As proved by experimental pushing tests performed by Railways Operators (DB, SNCF) [4], the normal force on the buffer heads, due to LCF between consecutive vehicles, and also the friction coefficient between the buffer heads influence significantly the derailment risk. The above experimental tests refer to a series of vehicles where the first performs a braking and the last a traction, in order to impose a constant compressive longitudinal forces on the test wagon running on an $\mathrm{S}$ shaped curve.

In the paper, the main mathematical models are shortly described. Then a comparison between simulated and experimental data is shown. Lastly the effects of some operational parameters, such as the buffers gap and the friction coefficient between the buffer heads, on running safety, are investigated.

\section{The numerical models}

\subsection{The TOAM wheel rail contact model}

In order to accurately reproduce the wheel-rail interaction forces, an on-line threedimensional contact model has been developed [1-3]; such model will be afterwards mentioned by using the acronyms TOAM (third order approximation method). General profiles of wheel and rail can be managed by the model and they can be defined from the user by input points; a mathematical definition of the profile is performed by employing the minimum number of third order polynomial functions that fit the input points with a tolerance defined by the user. The wheel and rail profiles used in the simulations proposed in this paper are respectively ORE 1002 and UIC 60 defined in $[5,6]$. These profiles are defined by 284 points for the wheel and 190 for the rail; the tolerance used to fit these points is $0.001 \mathrm{~mm}$, obtaining, respectively, 9 third order polynomial functions for the rail and 16 for the wheel.

The evaluation of contact points is based on the DIFF method [7-9], which has been modified, by introducing a numerical approximation of the wheel surface, in order to improve the time efficiency of contact points detection, by a forward analytical development of the mathematical model. This approximation does not influence significantly the accuracy of the contact point detection as described in [1]; particularly, a good accuracy is obtained (with an error on the conicity angle below $5 \%$, respect to commercial software), if the maximum relative angles between the surfaces of wheel and rail are enclose in the common domain for railway operations (experimentally they are lower than $20 \mathrm{mrad}$ for the attack angle and $33 \mathrm{mrad}$ for the roll angle, as it can be inferred by [4]) .

The previously mentioned approximation of the wheel surface consists in developing the wheel profile along a parabolic function that approximates the wheel circle in the lower point, as described in Figure 1. 


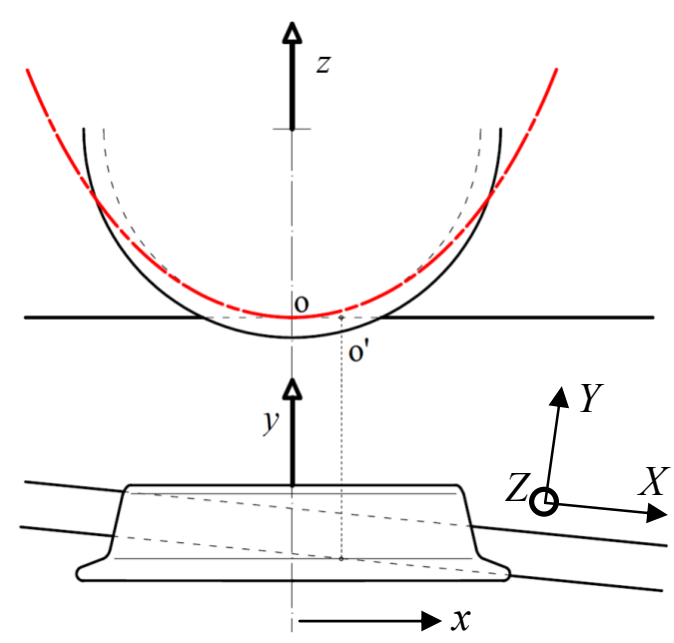

Figure 1: Wheel and rail reference system and wheel surface approximation.

Here, the track and the wheelset reference systems are indicated, respectively, by $\{X, Y, Z\}$ and $\{x, y, z\}$. The $Z$ axis is perpendicular to the track plane, the $X$ axis is oriented as the rail extrusion and the $Y$ axis is obtained by means of the right hand convention. The second reference system $\{x, y, z\}$ is located at the wheelset mass center, the $y$ axis is parallel to the wheelset axle, the $x$ axis is parallel to the track plane $(\mathrm{X}, \mathrm{Y})$ and the $z$ axis is obtained according to a right-hand reference system.

By employing the DIFF method, the contact points are detected, by looking for the lower points of the difference function, defined as the mathematical difference along the $\mathrm{Z}$ axis of the wheel and rail surfaces, as described in the Equation ( 1 ); where $\Sigma_{w}$ and $\Sigma_{r}$ are, respectively, the wheel and rail surfaces.

$$
\mathcal{D}=\Sigma_{w}(X, Y)-\Sigma_{r}(Y)
$$

According to the DIFF method, the contact points detection requires to find the minimum points of a two variable function. In order to reduce the computational effort, a further mathematical development is carried out, by introducing another necessary but no sufficient condition for the contact points detection. This condition imposes that the contact points must be located in a region of wheel surface where the derivative, computed along the direction of rail extrusion in the wheelset reference system, is null; such further condition provides a mathematical relation between $X(y)$ and $Y(y)$. Equation ( 1 ) can be rewritten in the Equation ( 2 ) and the contact points can be detected, by finding the minimum of a one variable equation; by this way the computational efficiency is improved and the on-line implementation is managed more easily.

$$
\mathcal{D}=\Sigma_{w}(y)-\Sigma_{r}(y)
$$

By knowing the position and the geometrical parameters at the contact points, the normal elastic contact forces are computed by using the Hertz theory [10]; moreover, the friction forces are computed by using the Polach friction model [11, $12]$. 


\subsection{The buffers contact model}

In order to consider the interaction forces exchanged by consecutive vehicles through their buffers, a new module has been added to the previously mentioned multibody code [3].

This new module has been developed by the Authors, in order to improve the corresponding previous model employed in TrainDy [13], which has been developed at the Universdity of Rome Tor Vergata with the financial support of Faiveley Transport of Italy and afterwards certified by the UIC [14]. The buffer contact model is now adapted and implemented in the multibody code.

The geometry of contact between the buffer heads is not still evaluated as a threedimensional contact but as a planar contact; in fact, in this study the offset between the buffer heights over the track plane between consecutive vehicles is not considered; but only the effect of normal and friction forces parallel to the track plane.

The forces exchanged by consecutive vehicles are evaluated by considering the geometry of buffers, in terms of their position on the vehicle and of head radii; moreover, the experimental load-unload elastic characteristic of buffers and draw gears defined by the user are employed. The numerical results, shown afterwards, are obtained by employing the experimental load-unload elastic characteristics of buffers and draw gear, taken by [4] Appendix 14 and 15, and displayed in Figure 2 and in Figure 3.

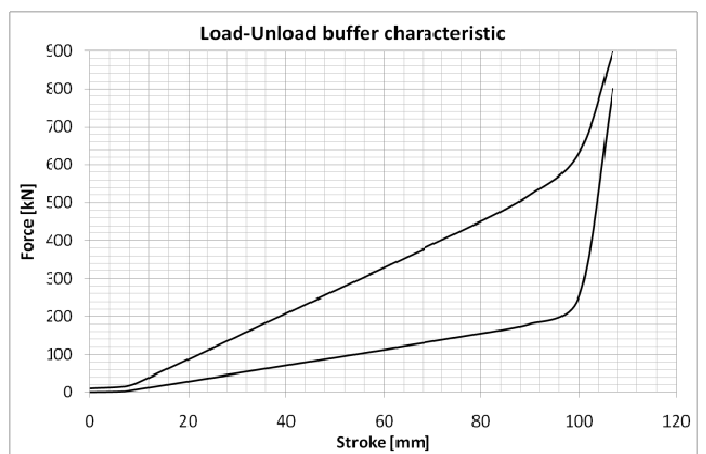

Figure 2: Buffer characteristic.

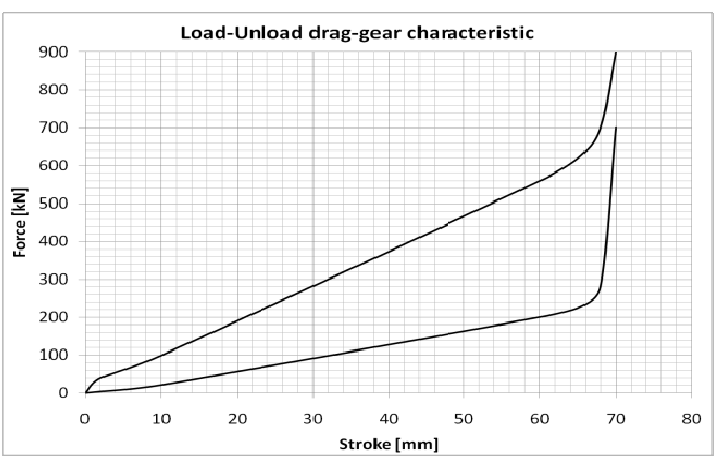

Figure 3: Draw gear characteristic.

From the force-displacement characteristics defined above, it is possible to compute the buffers and draw gears forces according to the model reported in [13].

The buffer head geometry here considered has a curvature radius of $1.5 \mathrm{~m}$, as specified in UIC Leaflet 527-1, point 1.4. 


\section{The vehicle dynamics in pushing tests}

\subsection{Test data}

In order to analyse the effects of the forces exchanged by buffers and draw gears, on vehicle dynamics, some pushing tests proposed in [4] are reproduced by means of the developed multibody code and the additional module introduced in the above paragraph. In particular, the effects of longitudinal compressing forces (LCF), of the gap and of the friction coefficient between the buffer heads are considered.

The simulated vehicle is a SNCF Gbs 254; the main geometrical characteristics of this vehicle, defined in [4] Appendix 4.1, are reported in Figure 4.

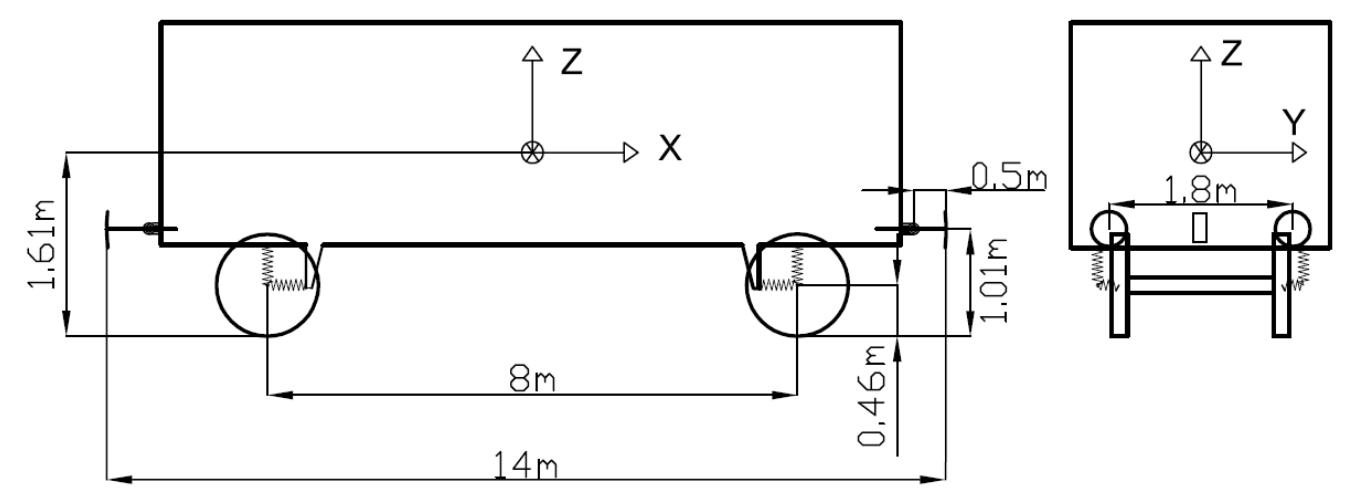

Figure 4: Gbs 254 wagon geometry.

The mass and inertia characteristics of the vehicle are reported in Table 1; moreover the elastic characteristics of the suspension are reported in Table 2.

\begin{tabular}{|ccccc|}
\hline & Mass $[\mathrm{kg}]$ & $\mathrm{I}_{\mathrm{xx}}\left[\mathrm{kg} \cdot \mathrm{m}^{2}\right]$ & $\mathrm{I}_{\mathrm{yy}}\left[\mathrm{kg} \cdot \mathrm{m}^{2}\right]$ & $\mathrm{I}_{\mathrm{zz}}\left[\mathrm{kg} \cdot \mathrm{m}^{2}\right]$ \\
\hline \hline Car body & 13300 & 32000 & 420000 & 413000 \\
Wheelset & 1490 & 988 & 90 & 988 \\
\hline
\end{tabular}

Table 1: Mass and inertia characteristics of the simulated wagon.

\begin{tabular}{|cccc|}
\hline & $\mathrm{X}$ & $\mathrm{Y}$ & $\mathrm{Z}$ \\
\hline \hline $\mathrm{K}[\mathrm{N} / \mathrm{m}]$ & $12 \cdot 10^{6}$ & $1.4 \cdot 10^{6}$ & $970 \cdot 10^{3}$ \\
$\mathrm{C}\left[\mathrm{N} \cdot \mathrm{s}^{2} / \mathrm{m}\right]$ & $12 \cdot 10^{4}$ & $1.8 \cdot 10^{5}$ & $640 \cdot 10^{3}$ \\
\hline
\end{tabular}

Table 2: Primary suspension characteristics.

The track gauge considered in the simulations is $1435 \mathrm{~mm}$; the wheel and rail profiles used are, respectively, the ORE S1002 and the UIC 60.

In the experimental tests reported in [4], nine consecutive vehicles are employed; the monitored test wagon is placed in the middle of the train. In order to impose a 
fixed value of longitudinal forces, the first vehicle performs a braking whereas the last vehicle, a locomotive, performs a controlled traction. The vehicles adjacent to the test wagon are a Tds on the front and an Rs wagon on the rear; these wagons are loaded whereas the test wagon is unloaded, in order to reduce the wheel lifting risk of the non-tested wagons. In the experimental tests, with the train placed on a straight track, the draw gears between the tested wagon and each one of the adjacent wagons were drawn, until the buffers were put in contact.

In order to reduce the computational effort and the complexity of the problem, only three equal consecutive vehicles are simulated. The central vehicle is the test vehicle and the adjacent vehicles are loaded with 30 tons, in order to avoid the wheel lifting. The external buffers of the adjacent vehicles are loaded with longitudinal forces along the $X$ axis of the car body (see Figure 4); by this way the compression of the train is imposed.

The track layout considered in the test is a $150 \mathrm{~m}$ track curve, with reverse curve and intermediate straight section, as it is specified in UIC Leaflet 527-1; this track layout is considered, according to the Railways Operators, as the most demanding from the point of view of running safety under a longitudinal compressive force.

The running speed used for the simulation is $30 \mathrm{~km} / \mathrm{h}$. Moreover, the experimental tests taken in account consider lubricated buffer heads; for this reason, a friction coefficient of 0.1 is considered in the numerical tests.

\subsection{Tests results}

In this paragraph, the numerical results are compared against experimental data; such data refer to a series of experimental tests carried on a family of wagons with characteristics similar to those reported above. In particular, in [4], several experimental tests are carried out considering similar two-axle vehicles in order to infer general safety rules. The differences, among the vehicles of this family, concern the stiffness of the primary suspensions, the stiffness of the axle journal, the torsional stiffness of the wagon and wheel base distance (some wagons have this parameters set to $9 \mathrm{~m}$, instead the simulated wagon has a wheel base distance equal to $8 \mathrm{~m}$ ). The other parameters, such as the wagon length (over buffers), the mass, the wheelset geometry and the buffers and draw gears elastic characteristics are the same for all the wagons of the family considered and are the same with respect to the simulated vehicle.

In order to define the operational limits, some criterions are taken into account as in [4]: the most limiting criterions for these tests are the "Distortion of journal axle" and the "Lateral displacement of the track".

According to [4], the "Distortion of journal axle" criterion prescribes that the distortion of the axle journal measured $380 \mathrm{~mm}$ below the lower flange of the solebar must not exceed $25 \mathrm{~mm}$; it corresponds to the limit of elastic deformation given in [4].

The "Lateral displacement of the track" criterion prescribes that the maximum lateral force applied to the track is function of the static axle load "P" (in $\mathrm{kN}$ ); for reinforced track the value can be computed by the Equation ( 3 ), according to [4]. 


$$
\left(H_{l i m}\right)_{2 m}=25+0.6 \bullet P=64[k N]
$$

The "Y/Q" criterion does not reach the prescribed limit $(Y / Q=1.2)$, in any experimental or simulated tests, i.e. according to this criterion, the vehicle is never in a critical condition.

In Figure 5, the maximum lateral force $F y_{2 m}$, computed by the developed simulator and applied to the track as function of the LCF, is reported, with a continuous line. The lateral force $F y_{2 m}$ is computed as an average on $2 \mathrm{~m}$, according to the prescribed criterion, above mentioned. The continuous line is obtained by using a friction coefficient between buffer heads of 0.1 ; this value is assumed valid for lubricated buffer heads. The experimental data are indicated by means of a circle and triangles: each point represents the mean value of a series of nominally equal tests, considering only the $95 \%$ of data close to the overall mean (i.e. discarding the tails of the original test distribution). The circle describes the results of several experimental tests performed employing a test wagon identical to the one simulated by the developed multibody code, whereas the triangles are the experimental results coming from the same family of wagons. By comparing the experimental data with the simulated counterpart, it is evident that the model proposed reproduces with a good accuracy the experimental behaviour.

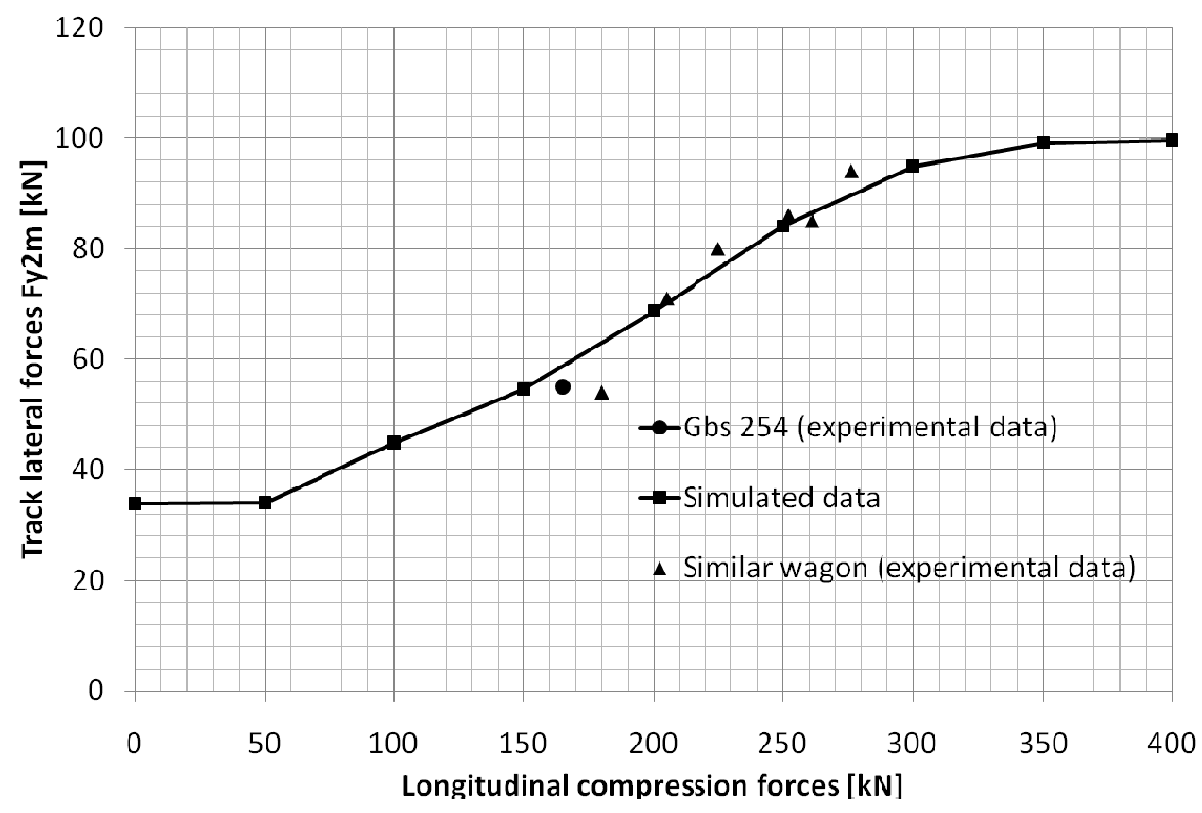

Figure 5: Maximum lateral force (over 2m) applied to the track as function of LCF.

By using the above mentioned criterions, it is possible to find the maximum admissible longitudinal forces for a given wagon, by means of the simulated and also experimental data. These values are summarized in Table 3, where, the results of experimental tests carried on the wagon Gbs 254 are reported. In each case, experimental or simulated, the "Distortion of the journal axle" criterion that leads in 
this case to a limit value of $25 \mathrm{~mm}$, has been reached before the "Lateral displacement of the track" criterion. When the first criterion is reached, the experimental and simulated lateral forces on the track $\left(F y_{2 m}\right)$ are, respectively, 55 and $61 \mathrm{kN}$, lower than the limiting value of $64 \mathrm{kN}$ defined by the second criterion (see Equation ( 3 )).

\begin{tabular}{c|cc}
\hline & Experimental & Simulated \\
\hline Longitudinal forces $[\mathrm{kN}]$ & $\mathbf{1 6 5}$ & $\mathbf{1 7 0}$ \\
Lateral force on the track $F y_{2 m}[\mathrm{kN}]$ & $\mathbf{5 5}$ & $\mathbf{6 0}$ \\
\hline
\end{tabular}

Table 3: Comparison of experimental and simulated safety limits, when the limit of the "Distortion of the axle journal" criterion is reached.

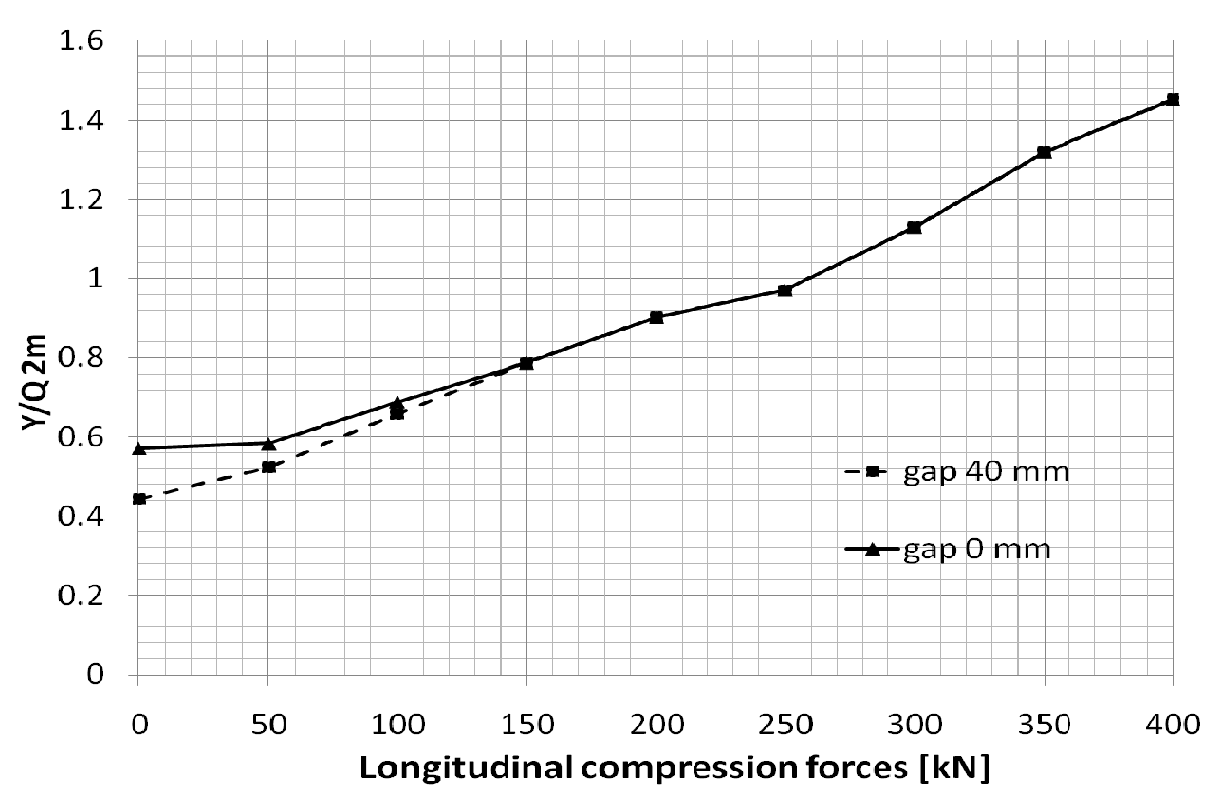

Figure 6: Maximum value of $Y / Q_{2 m}$ reached as function of the LCF and gap between buffer heads with lubricated surfaces.

Figure 6 shows the evolution of the $\mathrm{Y} / \mathrm{Q}_{2 \mathrm{~m}}$, computed with an average on $2 \mathrm{~m}$, as function of LCF and of the gap between buffers, considering a friction coefficient between the buffer heads of 0.1 (lubricated buffers). In this case, the $\mathrm{Y} / \mathrm{Q}_{2 \mathrm{~m}}$ parameter is not used to evaluate the safety limits, but in order to investigate the effect of the gap. The continuous line is computed considering $0 \mathrm{~mm}$ of gap, the wagons have been drawn until the buffers were put in contact, instead the dotted line shows the results when the wagon are drawn until the buffer heads have a gap of 40 $\mathrm{mm}$. In this figure it is clear that with a longitudinal compressive force less than 150 
$\mathrm{kN}$, the gap reduces the $\mathrm{Y} / \mathrm{Q}_{2 \mathrm{~m}}$ parameter. Such value of $150 \mathrm{kN}$ can be further explained by considering the Figure 7, showing the effect of gap between buffers; in this Figure 7, the maximum forces (averaged on $2 \mathrm{~m}$ ) acting on the buffers and on the draw gears are shown, as function of the externally imposed LCF, for different values of gap. When there is no LCF, due to the "bending" of the train on the curve, there are tensile forces at draw gears, caused by the relative angle among consecutive wagons, imposed by the curve, and therefore compressive forces at buffers arise. The gap reduces such forces, as shown by Figure 7; in both cases, the reported forces are highly affected by vehicle dynamic. By increasing the LCF, the draw gear tension is reduced, due to the track curve, hence the maximum forces of buffers compression become similar to the applied LCF and the advantage of the gap disappears (see the behaviour of the curves above $150 \mathrm{kN}$ ), as it has been already shown in Figure 6.

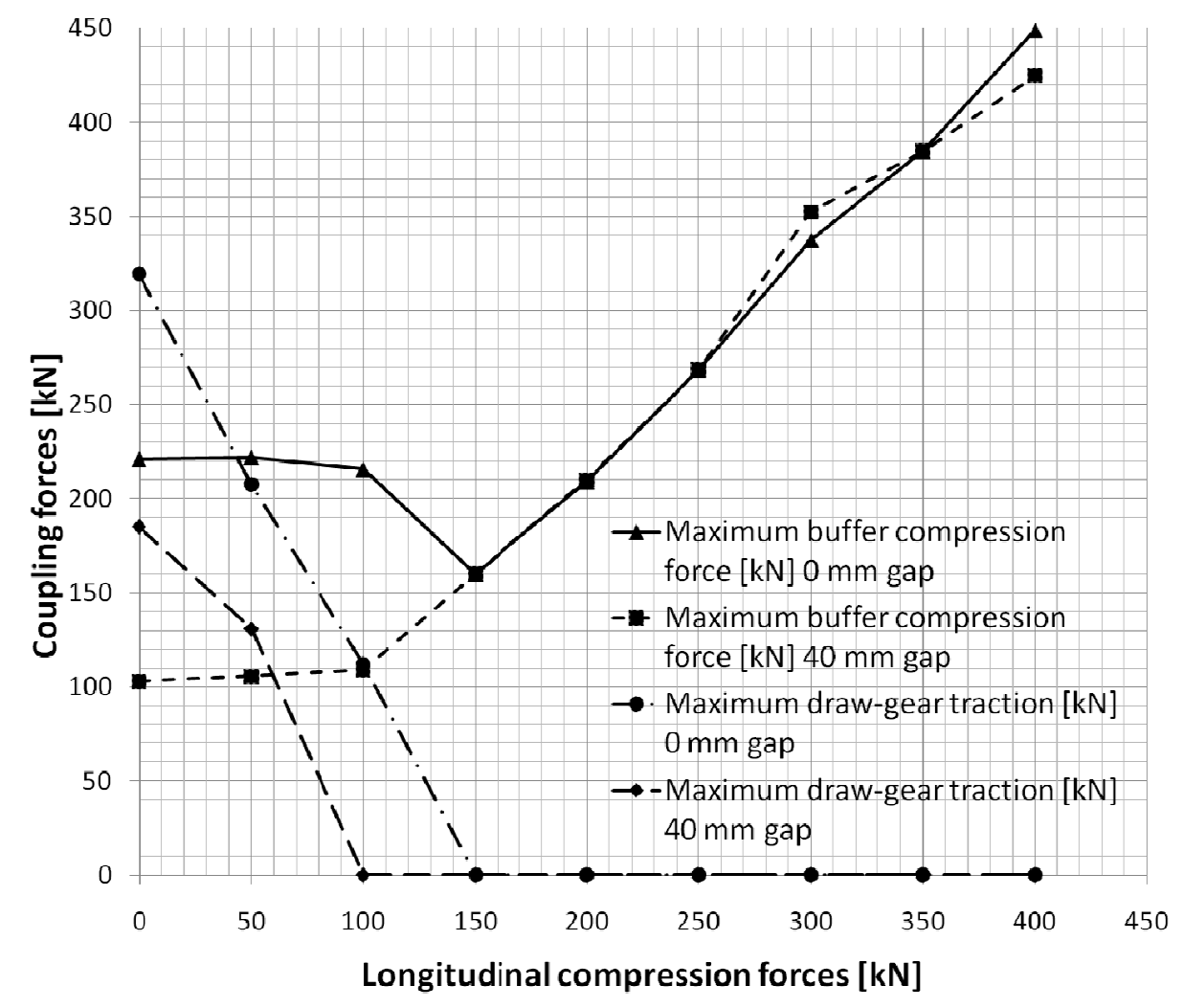

Figure 7: Maximum buffer compression forces and draw gear traction in function of the imposed LCF and gap between buffer heads.

The effect of gap and friction coefficient between buffer heads, when no LCF are applied, can be investigated by means of the parameter $F y_{2 m}$, as shown in Figure 8. It is clear that an increment of the friction coefficient between buffer heads brings a significant increment of the lateral force applied to the track; also in this case the $F y_{2 m}$ force can be reduced by using a gap between buffers especially for high friction coefficient. 


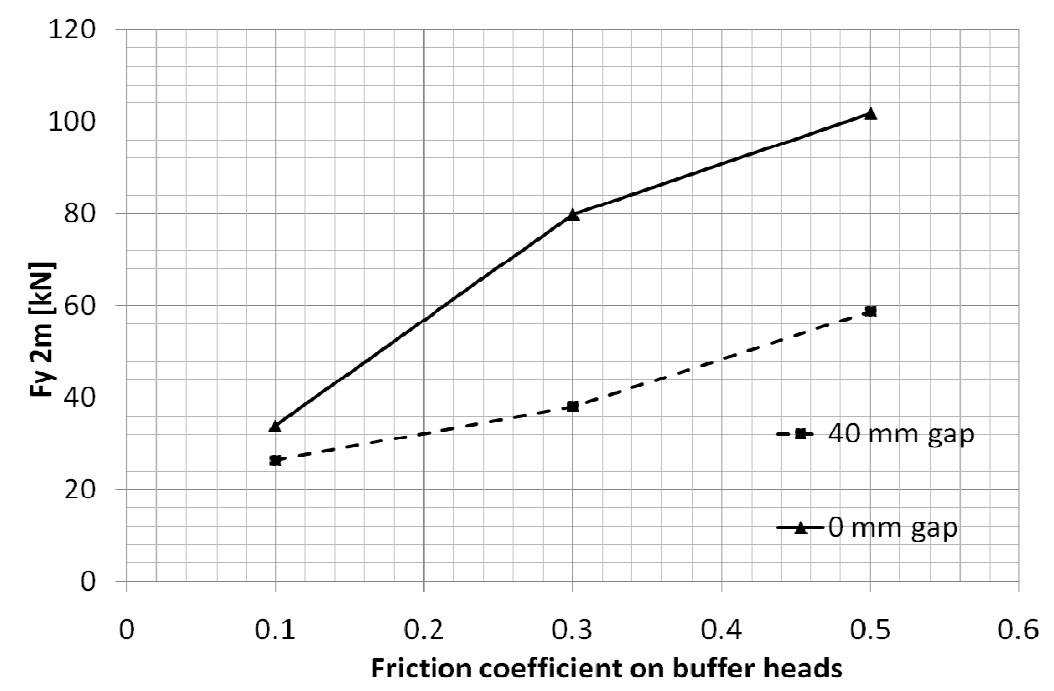

Figure 8: Maximum lateral force exchanged with the track (averaged on $2 \mathrm{~m}$ ), without LCF imposed, as function of the gap and friction coefficient between the buffer heads.

The beneficial effect of the gap, described above can be considered valid only for the same test condition of the test proposed, i.e. vehicle pushed by constant longitudinal compressive force on a curve. As a matter of fact, the presence of gap increases the LCF during a typical braking manoeuvre, due to the train longitudinal dynamics. In the test cases reported here, the train longitudinal dynamics is not highly excited due to the constant LCF imposed.

\section{Conclusions}

This paper shows the effects on vehicle dynamics of the forces exchanged by consecutive vehicles by means of buffers and draw gears, on a S shaped curve. The simulations are carried out by using a multibody code developed by the Authors, where each body is characterized by 6 degree of freedom and the wheel rail contact is managed three dimensionally by means of the contact model TOAM.

Experimental pushing tests on an "S" curve, concerning two axle vehicles, are reproduced in order to verify the model proposed and to perform preliminary analyses on the effects of buffers operational parameters, as the gap and the friction between the buffer heads.

Simulation results show that buffers compression forces occur also without externally imposed LCF due to the draw gears tension, when the wagons are on a curve. These forces increase the $Y / Q_{2 m}$ or $F y_{2 m}$ and they can be reduced by imposing a gap between the buffer heads. Moreover, the friction between buffer heads considerably increase the maximum lateral forces applied to the track. 


\section{References}

[1] D. Negretti, "A third order approximation method for three-dimensional wheel rail contact", Vehicle System Dynamics, 2011. (accepted)

[2] L. Cantone, D. Negretti, V. Vullo "Sviluppo e validazione di un modello semianalitico del terz'ordine per l'analisi del contatto ruota rotaia", SEF 2011, Roma, Italy, 18 February, 2011.

[3] L. Cantone, D. Negretti, V. Vullo "Analisi del contatto ruota rotaia mediante un'approssimazione del terzo ordine", AIAS 2010, Maratea, Italy, 7-10 September, 2010.

[4] ORE Question B 12, Report No. 40, "Propelling tests with long two-axle wagons", UTRECHT, April 1984.

[5] S. Iwnick, "Manchester Benchmarks for Rail Vehicle Simulation", Vehicle System Dynamics, 30/3-4, 295 - 313, 1998.

[6] S. Iwnick, "The Manchester Benchmarks for Rail Vehicle Simulation", Taylor \& Francis, ISSN 0042-4114

[7] J. Auciello, S. Falomi, M. Malvezzi, E. Meli, and P. Toni, Determination of wheel/rail contact points in the simulation of a railway vehicle dynamics. Springer Science+Business Media B.V., 2008.

[8] S. Falomi, M. Malvezzi, E. Meli, and M. Rinchi, Multibody modeling of railway vehicles: innovative algorithms for the detection of wheel-rail contact points. 2009, 8th International Conference on Contact Mechanics and Wear of Rail/Wheel Systems (CM2009), Firenze, Italy.

[9] A. A. Shabana, K. E. Zaazaa, and H. Suguyama, Railroad Vehicle Dynamics A computational approach. CRC Press, Taylor \& Francis Group, New York, 2007.

[10] S. Timoshenko and J. N. Goodier, Theory of Elasticity. McGraw-Hill Book Company, New York, 1951.

[11] O. Polach, A fast wheel-rail forces calculation computer code. Vehicle System Dynamic Supplement, 33, 1999, 728-739.

[12] O. Polach, Influence of Locomotive Tractive Effort on the Forces Between Wheel and Rail. Vehicle System Dynamic Supplement, 35, 2001, 7-22.

[13] L. Cantone, D. Negretti, "Modellazione dinamica disaccoppiata dei respingenti ferroviari", AIAS 2009 9-11 Settembre Torino, 2009.

[14] L. Cantone, "TrainDy: the new Union Internationale des Chemins de Fer software for freight train interoperability", Proc. IMechE, Part F: J. Rail and Rapid Transit, 2011, 225 (F1), 57-70. DOI 10.1243/09544097JRRT347. 